Georgian Mathematical Journal

Volume 13 (2006), Number 3, 411-417

\title{
CURVES WITH TWO PENCILS AND ASSOCIATED MAPS TO PROJECTIVE SPACES
}

\author{
EDOARDO BALLICO
}

\begin{abstract}
Let $X$ be a smooth and connected projective curve. Assume the existence of spanned $L \in \operatorname{Pic}^{a}(X), R \in \operatorname{Pic}^{b}(X)$ such that $h^{0}(X, L)=$ $h^{0}(X, R)=2$ and the induced map $\phi_{L, R}: X \rightarrow \mathbf{P}^{1} \times \mathbf{P}^{1}$ is birational onto its image. Here we study the following question. What can be said about the morphisms $\beta: X \rightarrow \mathbf{P}^{r}$ induced by a complete linear system $\left|L^{\otimes u} \otimes R^{\otimes v}\right|$ for some positive $u, v$ ? We study the homogeneous ideal and the minimal free resolution of the curve $\beta(X)$.
\end{abstract}

2000 Mathematics Subject Classification: 14H51, 14H50.

Key words and phrases: Maps to projective spaces, line bundles on curves, curves with two pencils, homogeneous ideal, minimal free resolution.

\section{The Statements}

A very classical problem (studied at least since C. Segre and G. Castelnuovo) is the study of the homogeneous ideal of curves embedded in a projective space. The top result of the classical period was K. Petri's analysis of the homogeneous ideals of all canonically embedded curves (1924). Of course, this was related to two fundamental papers of D. Hilbert (1890 and 1893). As far as we know, the modern period began with D. Mumford ([5]), which made clear to everybody the geometric significance of knowing the minimal free resolution of an embedded variety. The main breakthrough came with M. Green's paper [4], in which he gave several tools to compute syzygies and introduced the definition of property $N_{p}, p \geq 0$. It has been clear since the classical study of the homogeneous ideals of canonically embedded trigonal curves, that if an embedded curve $Y \subset \mathbf{P}^{n}$ is contained in a low degree surface, then its minimal free resolution is very restricted (it cannot be "very nice", but much of it can be explicitly computed). Here we try to apply this obvious remark to certain morphisms $\phi: X \rightarrow \mathbf{P}^{n}, X$ a smooth curve, which are not embeddings. Let $X$ be a smooth and connected projective curve of genus $g$. Assume the existence of spanned $L \in \operatorname{Pic}^{a}(X), R \in$ $\operatorname{Pic}^{b}(X)$ such that $h^{0}(X, L)=h^{0}(X, R)=2$ and the induced map $\phi_{L, R}: X \rightarrow$ $\mathbf{P}^{1} \times \mathbf{P}^{1}$ is birational onto its image. What can be said about the morphisms $X \rightarrow \mathbf{P}^{r}$ induced by a complete linear system $\left|L^{\otimes u} \otimes R^{\otimes v}\right|$ for some positive $u, v ?$

We work over an algebraically closed field $\mathbb{K}$. In our first result we make a strong assumption: the genus $g$ must be very near to $p_{a}\left(\phi_{L, R}(X)\right)=a b-a-b+1$. We do not make other assumptions on $X$. 
Theorem 1. Fix integers $a, b, g, u, v$ such that $a \geq 3, b \geq 3,1 \leq u \leq a-2$, $1 \leq v \leq b-2$, and $a b-a-b+1-\min \{a-1-u, b-1-v\} \leq g \leq a b-a-b+1$. Let $x$ be a minimal integer such that $a \leq x u$ and $b \leq x v$. Let $X$ be a smooth and connected curve of genus $g$ such that there are base point free $L \in \operatorname{Pic}^{a}(X)$, $R \in \operatorname{Pic}^{b}(X)$ such that $h^{0}(X, L)=h^{0}(X, R)=2$ and the induced map $\phi_{L, R}$ : $X \rightarrow \mathbf{P}^{1} \times \mathbf{P}^{1}$ is birational onto its image. Set $M:=L^{\otimes u} \otimes R^{\otimes v}$. Then:

(i) $h^{0}(X, M)=(u+1)(v+1)$, the associated morphism $\phi_{M}: X \rightarrow \mathbf{P}^{r}$, $r:=u v+u+v$, is birational onto its image and $Y:=\phi_{M}(X) \cong C:=$ $\phi_{L, R}(X)$.

(ii) $Y$ is arithmetically normal and the homogeneous ideal $I(Y)(*)$ of $Y$ in $\mathbf{P}^{r}$ is generated by forms of degree at most $x$;

(iii) if $x=2$, then $Y$ has Property $\mathrm{N}_{1}$ (i.e., it is arithmetically CohenMacaulay and its homogeneous ideal is generated by quadrics) and $h^{0}\left(\mathbf{P}^{r}, \mathcal{I}_{Y}(2)\right)=(u v+u+v+2)(u v+u+1) / 2-(2 u+1)(2 v+1)+(2 u+$ $1-a)(2 v+1-b)$;

(iv) if $x \geq 3$, then $h^{0}\left(\mathbf{P}^{r}, \mathcal{I}_{Y}(2)\right)=(u v+u+v+2)(u v+u+1) / 2-(2 u+$ 1) $(2 v+1)$ and $I(Y)(*)$ is minimally generated by $(u v+u+v+2)(u v+u+$ $1) / 2-(2 u+1)(2 v+1)$ forms of degree 2 and $(x u-a+1)(x v-b+1)$ forms of degree $x$; furthermore, the base locus of $H^{0}\left(\mathbf{P}^{r}, \mathcal{I}_{Y}(2)\right)$ is projectively equivalent to the embedding of $\mathbf{P}^{1} \times \mathbf{P}^{1}$ by the complete linear system $\left|\mathcal{O}_{\mathbf{P}^{1} \times \mathbf{P}^{1}}(u, v)\right|$.

Remark 1 . Use the set-up of Theorem 1 , but in the omitted case $g=a b-$ $a-b+1, u=a$ and $v=b$. In this case $\phi_{L, R}$ is an isomorphism onto its image $C$ and $C$ is a hyperplane section of the embedding of $\mathbf{P}^{1} \times \mathbf{P}^{1}$ induced by the complete linear system $\left|\mathcal{O}_{\mathbf{P}^{1} \times \mathbf{P}^{1}}(a, b)\right|$. By [3], Theorem 1.3, $M$ has Property $\mathrm{N}_{2 a+2 b-3}$, but not Property $\mathrm{N}_{2 a+2 b-2}$.

Remark 2. Assume char $(\mathbb{K})=0$. Fix integers $g, a, b$ such that $a \geq 5$ and $b \geq 8$. Let $S(g ; a, b)$ denote the constructible subset of $\mathcal{M}_{g}$ parametrizing all smooth genus $g$ curves having a morphism $\phi: X \rightarrow \mathbf{P}^{1} \times \mathbf{P}^{1}$ with image of type $(a, b)$ and which is birational onto its image. By [1], Theorem 0.1 and 0.2 , $S(g ; a, b)$ is irreducible and $\operatorname{dim}(S(g ; a, b))=a b+a+b-(a b-a-b+1-g)-8=$ $2 a+2 b+g-9$.

To state our next result we introduce the following notation.

Notation 1. For all integers $a \geq 2, b \geq 2, x \geq 0, y \geq 0$ and $s$ such that $0 \leq s \leq a b-a-b+1$ and any algebraically closed field $\mathbb{K}$ we will say that $\wp(\mathbb{K} ; a, b, x, y, s)$ is true if there is an integral nodal curve $C$ of type $(a, b)$ on $\mathbf{P}^{1} \times \mathbf{P}^{1}$ such that $\sharp\left(\operatorname{Sing}(C)=s\right.$ and $h^{0}\left(\mathbf{P}^{1} \times \mathbf{P}^{1}, \mathcal{I}_{\operatorname{Sing}(C)}(x, y)\right)=$ $\max \{(x+1)(y+1)-s, 0\}$, i.e., such that $h^{1}\left(\mathbf{P}^{1} \times \mathbf{P}^{1}, \mathcal{I}_{\operatorname{Sing}(C)}(x, y)\right)=\max \{s-$ $(x+1)(y+1), 0\}$.

Obviously, $\wp(\mathbb{K} ; a, b, x, y, s)$ and $\wp(\mathbb{L} ; a, b, x, y, s)$ are equivalent if char $(\mathbb{K})=$ $\operatorname{char}(\mathbb{L})$. If $\operatorname{char}(\mathbb{K})=0$, then the corresponding property in $\mathbf{P}^{2}$ is true $([6]$, Anhang F, or [9], Proposition 3.1). 
Theorem 2. Fix integers $a, b, g, u, v$ such that $a \geq 3, b \geq 3,1 \leq u \leq a-2$, $1 \leq v \leq b-2$ and $a b-a-b-(a-2-u)(b-2-u)+a+b-u-v+4 \leq g \leq$ $a b-a-b+1$. Assume $\wp(\mathbb{K} ; a, b, a-2-u, b-2-v, a b-a-b+1-g)$. Let $x$ be a minimal integer such that $a \leq x u$ and $b \leq x v$. There are a smooth and connected curve $X$ of genus $g$ and base point free $L \in \operatorname{Pic}^{a}(X), R \in \operatorname{Pic}^{b}(X)$ such that $h^{0}(X, L)=h^{0}(X, R)=2$, the induced map $\phi_{L, R}: X \rightarrow \mathbf{P}^{1} \times \mathbf{P}^{1}$ is birational onto its image, $C:=\phi_{L, R}(X)$ is nodal, $p_{a}(C)=a b-a-b+1$, and the spanned line bundle $M:=L^{\otimes u} \otimes R^{\otimes v}$ on $X$ has the following properties:

(i) $h^{0}(X, M)=(u+1)(v+1)$, the associated morphism $\phi_{M}: X \rightarrow \mathbf{P}^{r}$, $r:=u v+u+v$, is birational onto its image and $Y:=\phi_{M}(X) \cong C$.

(ii) $Y$ is arithmetically normal and the homogeneous ideal $I(Y)(*)$ of $Y$ in $\mathbf{P}^{r}$ is generated by forms of degree at most $x$;

(iii) if $x=2$, then $Y$ has Property $\mathrm{N}_{1}$ and $h^{0}\left(\mathbf{P}^{r}, \mathcal{I}_{Y}(2)\right)=(u v+u+v+$ $2)(u v+u+1) / 2-(2 u+1)(2 v+1)+(2 u+1-a)(2 v+1-b)$;

(iv) if $x \geq 3$, then $h^{0}\left(\mathbf{P}^{r}, \mathcal{I}_{Y}(2)\right)=(u v+u+v+2)(u v+u+1) / 2-(2 u+$ $1)(2 v+1)$ and $I(Y)(*)$ is minimally generated by $(u v+u+v+2)(u v+u+$ $1) / 2-(2 u+1)(2 v+1)$ forms of degree 2 and $(x u-a+1)(x v-b+1)$ forms of degree $x$; furthermore, the base locus of $H^{0}\left(\mathbf{P}^{r}, \mathcal{I}_{Y}(2)\right)$ is projectively equivalent to the embedding of $\mathbf{P}^{1} \times \mathbf{P}^{1}$ by the complete linear system $\left|\mathcal{O}_{\mathbf{P}^{1} \times \mathbf{P}^{1}}(u, v)\right|$.

Remark 3. By Lemma 2 below $\wp(\mathbb{K} ; a, b, u, v, a b-a-b+1-g)$ is true if $a b-a-b+1-g \leq u v-u-v+1$ and $v \leq a, u \leq a$. Hence we may drop the corresponding assumption in the statement of Theorem 2 if we assume $1 \leq u \leq \min \{a-2, b\}$ and $1 \leq v \leq \min \{b-2, a\}$.

When $x=2$ we will also look at the minimal free resolution of $Y$. In section 3 we will prove the following result.

Theorem 3. Assume char $(\mathbb{K})=0$. Fix integers $a, b, g, u, v$ such that $3 \leq$ $u+2 \leq a \leq 2 u, 3 \leq v+2 \leq b \leq 2 v$, and $a b-a-b-(a-2-u)(b-2-u)+$ $a+b-u-v+4 \leq g \leq a b-a-b+1$. Take $Y \subset \mathbf{P}^{r}, r:=u v+u+v$, as in the statement of Theorem 1 for $x=2$. Then $Y$ has Property $N_{i}$ for all integers $i$ such that $0 \leq i \leq \min \{u, v, 2 u-b, 2 v-a\}$.

\section{The Proofs of Theorems 1 And 2}

Lemma 1. Fix integers $a \geq 2, b \geq 2, x \geq 2, k>0, t \geq x, u, v$ such that $0<u \leq a \leq x u-1$ and $0<v \leq b \leq x v-1$, and an integral curve $C \in$ $\left|\mathcal{O}_{\mathbf{P}^{1} \times \mathbf{P}^{1}}(a, b)\right|$. Then the restriction map $\rho_{C, t}: H^{0}\left(\mathbf{P}^{1} \times \mathbf{P}^{1}, \mathcal{O}_{\mathbf{P}^{1} \times \mathbf{P}^{1}}(t u, t v)\right) \rightarrow$ $H^{0}\left(C, \mathcal{O}_{C}(t u, t v)\right)$ is surjective and the natural multiplication map $\eta_{C, t, k}$ : $S^{k}\left(H^{0}\left(\mathbf{P}^{1} \times \mathbf{P}^{1}, \mathcal{O}_{\mathbf{P}^{1} \times \mathbf{P}^{1}}(k u, k v)\right)\right) \otimes \operatorname{Ker}\left(\rho_{C, t}\right) \rightarrow \operatorname{Ker}\left(\rho_{C, t+k}\right)$ is surjective.

Proof. The first assertion follows from $h^{1}\left(\mathbf{P}^{1} \times \mathbf{P}^{1}, \mathcal{O}_{\mathbf{P}^{1} \times \mathbf{P}^{1}}(t u-a, t v-b)\right)=0$, which is true because $t \geq x$ and hence $t u-a \geq-1$ and $t v-b \geq-1$. By induction on $k$ we reduce the second assertion to the case $k=1$. Use that 
$C \in\left|\mathcal{O}_{\mathbf{P}^{1} \times \mathbf{P}^{1}}(a, b)\right|$ and the surjectivity of the multiplication map

$$
\begin{aligned}
H^{0}\left(\mathbf{P}^{1}\right. & \left.\times \mathbf{P}^{1}, \mathcal{O}_{\mathbf{P}^{1} \times \mathbf{P}^{1}}(u, v)\right) \otimes H^{0}\left(\mathbf{P}^{1} \times \mathbf{P}^{1}, \mathcal{O}_{\mathbf{P}^{1} \times \mathbf{P}^{1}}(t u-a, t v-b)\right) \\
& \rightarrow H^{0}\left(\mathbf{P}^{1} \times \mathbf{P}^{1}, \mathcal{O}_{\mathbf{P}^{1} \times \mathbf{P}^{1}}((t+1) u-a,(t+1) v-b)\right) .
\end{aligned}
$$

Lemma 2. Fix integers $a \geq 2, b \geq 2,0 \leq s \leq a b-a-b+1,0 \leq x \leq b$ and $0 \leq y \leq a$. Then there exists an integral nodal curve $C \subset \mathbf{P}^{1} \times \mathbf{P}^{1}$ such that $C$ has type $(a, b), \sharp(S)=s, h^{0}\left(\mathbf{P}^{1} \times \mathbf{P}^{1}, \mathcal{I}_{S}(x, y)\right)=\max \{0,(x+1)(y+1)-s\}$ and $h^{1}\left(\mathbf{P}^{1} \times \mathbf{P}^{1}, \mathcal{I}_{S}(x, y)\right)=\max \{0, s-(x+1)(y+1)\}$, where $S:=\operatorname{Sing}(C)$.

Proof. Set $\tilde{s}:=(a-1)(b-1)$. Let $T_{i} \subset \mathbf{P}^{1} \times \mathbf{P}^{1}, 1 \leq i \leq a$, be a general curves of type $(1,0)$ and $D_{j} \subset \mathbf{P}^{1} \times \mathbf{P}^{1} b$ general curves of type $(0,1)$. Set $Y:=$ $\bigcup_{i=1}^{a} T_{i} \cup \bigcup_{j=1}^{b} D_{j}$. Hence $Y$ is a nodal curve of type $(a, b)$ with $a+b$ irreducible components, all of them being smooth and rational. Set $A:=\operatorname{Sing}(Y)$. First assume $y \geq a-2$. Set $B:=\cup_{i=2}^{a} \cup_{j=2}^{b} T_{i} \cap T_{j}$. Hence $\sharp(B)=(a-1)(b-1)=p_{a}(Y)$. Call $B$ the unassigned nodes of $Y$. Since the dual of $\omega_{\mathbf{P}^{1} \times \mathbf{P}^{1}}$ is ample, we may apply [8], Lemma 2.2, and obtain the existence of a family $\left\{C_{\lambda}\right\}_{\lambda \in \Lambda}$ of integral nodal curves of type $(a, b)$ which smoothes exactly the unassigned nodes of $Y$. Notice that $Y \backslash B$ is connected, i.e., the pair $(Y, B)$ is virtually connected in the sense of [8]. By semicontinuity, in this case we may take $C=C_{\lambda}$ for general $\lambda \in \Lambda$. Now assume $s<\tilde{s}$. We make the previous construction taking $\tilde{s}-s$ more unassigned nodes and again apply [8], Lemma 2.2.

Proofs of Theorems 1 and 2. For both theorems $C$ is an integral curve of type $(a, b)$ on $\mathbf{P}^{1} \times \mathbf{P}^{1}$. Thus $C$ is Gorenstein, $\omega_{C} \cong \mathcal{O}_{C}(a-2, b-2)$ and $p_{a}(C)=a b-a-b+1$. Since $C$ is Gorenstein, the conductor of $C$ in $X$ has length $p_{a}(C)-g$. Obviously, $M$ is spanned and birational onto its image. Let $\psi:=\mathbf{P}^{1} \times \mathbf{P}^{1} \rightarrow \mathbf{P}^{r}, r:=u v+u+v$, be the embedding associated to the complete linear system $\left|\mathcal{O}_{\mathbf{P}^{1} \times \mathbf{P}^{1}}(u, v)\right|$. Since the homogeneous ideal of $\psi\left(\mathbf{P}^{1} \times \mathbf{P}^{1}\right)$ is arithmetically Cohen-Macaulay and generated by quadrics (see [3] for much more), it is generated by $(u v+u+v+2)(u v+u+v+1) / 2-(2 u+1)(2 v+1)$ linearly independent quadrics. Notice that $\langle\psi(C)\rangle=\mathbf{P}^{r}$ because $u<a$ and $v<b$. The surface $\psi\left(\mathbf{P}^{1} \times \mathbf{P}^{1}\right)$ has Property $\mathrm{N}_{2 u+2 v-3}([3])$ and in particular it is projectively normal and its homogeneous ideal $I(\psi)(*)$ is generated by quadrics. Since $h^{1}\left(\mathbf{P}^{1} \times \mathbf{P}^{1}, \mathcal{O}_{\mathbf{P}^{1} \times \mathbf{P}^{1}}(t u, t v)\right)=0$ for all $t \in \mathbb{Z}$, the surface $\psi\left(\mathbf{P}^{1} \times \mathbf{P}^{1}\right)$ is also arithmetically Cohen-Macaulay. Since $u<a$ and $v<b$, the homogeneous ideal $I(\psi(C))(*)$ is obtained from $I(\psi)(*)$ adding some more hypersurphaces exactly as in parts (iii) and (iv) of both theorems. To conclude it is sufficient to prove that $h^{0}(X, M)=(u+1)(v+1)$ and $\phi_{M}(X)=\psi(C)$. Indeed, since $\psi(C)$ is a linear projection of $\phi_{M}(X)$, it is sufficient to prove $h^{0}(X, M)=(u+1)(v+1)$. By Riemann-Roch this equality is equivalent to the equality $h^{1}(X, M)=h^{1}\left(C, \mathcal{O}_{C}(u, v)\right)-p_{a}(C)+g$. By adjunction theory and duality we have $h^{1}\left(C, \mathcal{O}_{C}(u, v)\right)=h^{0}\left(C, \mathcal{O}_{C}(a-2-u, b-2-u)\right)=h^{0}\left(\mathbf{P}^{1} \times\right.$ $\left.\mathbf{P}^{1}, \mathcal{O}_{\mathbf{P}^{1} \times \mathbf{P}^{1}}(a-2-u, b-2-v)\right)$. Since $\mathbf{P}^{1} \times \mathbf{P}^{1}$ is a smooth surface, $M \cong$ $\phi_{L, R}{ }^{*}\left(\boldsymbol{I}^{1} \times \mathbf{P}^{1}(u, v-v)\right), h^{i}\left(\mathbf{P}^{1} \times \mathbf{P}^{1}, \omega_{\mathbf{P}^{1} \times \mathbf{P}^{1}}\right)=0$ for $i=1,2$, adjunction theory gives the existence of a length $p_{a}(C)-g$ zero-dimensional scheme $Z \subset \mathbf{P}^{1} \times \mathbf{P}^{1}$ such that $h^{1}(X, M)=h^{1}\left(C, \mathcal{O}_{C}(u, v)-h^{0}\left(\mathbf{P}^{1} \times \mathbf{P}^{1}, \mathcal{I}_{Z}(a-2-u, b-2-v)+\right.\right.$ 
$h^{0}\left(\mathbf{P}^{1} \times \mathbf{P}^{1}, \mathcal{O}_{\mathbf{P}^{1} \times \mathbf{P}^{1}}(a-2-u, b-2-v)\right)$; if $C$ is nodal, then $Z=\operatorname{Sing}(C)$ with its reduced structure. Thus it is sufficient to show that $Z$ imposes $a b-a-b+1-g$ independent conditions on $H^{0}\left(\mathbf{P}^{1} \times \mathbf{P}^{1}, \mathcal{O}_{\mathbf{P}^{1} \times \mathbf{P}^{1}}(a-2-u, b-2-v)\right)$. In the setup of Theorem 1 this is true for any zero-dimensional scheme with that length because $a b-a-b+1 \leq \min \{a-1-u, b-1-v\}$. In the set-up of Theorem 2 this is exactly the assumption that $\wp(\mathbb{K} ; a, b, a-2-u, b-2-v, a b-a-b+1-g)$ is true and the choice of the nodal curve $C$.

\section{The Minimal Free Resolution}

Here we prove Theorem 3 using Bott's vanishing theorem ([2]). Fix integers $a, b, g, u, v$ such that $3 \leq u+2 \leq a \leq 2 u, 3 \leq v+2 \leq b \leq 2 v, a b-a-$ $b-(a-2-u)(b-2-u)+a+b-u-v+4 \leq g \leq a b-a-b+1$, and set $r:=u v+u+v$. Let $\psi: \mathbf{P}^{1} \times \mathbf{P}^{1} \rightarrow \mathbf{P}^{r}$ and $C:=\psi(Y)$ be respectively the map and the integral curve introduced in the statement of Theorem 2. Set $\Sigma=\Sigma_{u, v}:=\psi\left(\mathbf{P}^{1} \times \mathbf{P}^{1}\right) \subset \mathbf{P}^{r}$. In the range of integers we will be interested in the minimal free resolution of the curve $C \subset \mathbf{P}^{r}$ (resp. surface $\Sigma \subset \mathbf{P}^{r}$ ) is "essentially determined" by the cohomology groups $h^{1}\left(\mathbf{P}^{r}, \mathcal{I}_{C} \otimes \Omega_{\mathbf{P}^{r}}(i+t)\right)$, $t \geq 2$, (resp. $\left.h^{1}\left(\mathbf{P}^{r}, \mathcal{I}_{\Sigma} \otimes \Omega_{\mathbf{P}^{r}}^{i}(i+t)\right), t \geq 2\right)$ and/or by the restriction maps $\rho_{C, t, i}: H^{0}\left(\mathbf{P}^{r}, \Omega_{\mathbf{P}^{r}}^{i}(i+t)\right) \rightarrow H^{0}\left(C, \Omega_{\mathbf{P}^{r}}^{i}(i+t) \mid C\right)\left(\operatorname{resp} . \rho_{C, t, i}: H^{0}\left(\mathbf{P}^{r}, \Omega_{\mathbf{P}^{r}}^{i}(i+\right.\right.$ $t)) \rightarrow H^{0}\left(\Sigma, \Omega_{\mathbf{P}^{r}}^{i}(i+t) \mid \Sigma\right)$. Set $E_{i}:=\Omega_{\mathbf{P}^{r}}^{i}(i) \mid \Sigma$. Let $u_{C, t}: H^{0}\left(\Sigma, E_{i}(t)\right) \rightarrow$ $H^{0}\left(C, E_{i}(t) \mid C\right)$ denote the restriction map. Hence $\rho_{C, t, i}=u_{C, t} \circ \rho_{\Sigma, t, i}$. We will identify $\Sigma$ with $\mathbf{P}^{1} \times \mathbf{P}^{1}$ as an abstract surface. In particular we will use the notation $\mathcal{O}_{\Sigma}(m, n)$ for the line bundle of type $(m, n)$. Hence $\mathcal{O}_{\Sigma}(1) \cong \mathcal{O}_{\Sigma}(u, v)$.

Remark 4. Assume char $(\mathbb{K})=0$. By the second part of [3], Theorem 1.3, $\Sigma$ has Property $N_{p}$ if and only if $p \leq 2 u+2 v-3$. Hence $\rho_{\Sigma, 2, i}$ is surjective for all $i \leq 2 u+2 v-3$.

Remark 5. Fix integers $i, t$ such that $h^{1}\left(\Sigma, E_{i}(t)\right)=0$. Then $\operatorname{Coker}\left(u_{C, t}\right) \cong$ $H^{1}\left(\Sigma, \mathcal{I}_{C} \otimes E_{i}(t)\right) \cong H^{1}\left(\Sigma, E_{i}(t u-a, t v-b)\right)$ and $\operatorname{Ker}\left(u_{C, t}\right) \cong H^{0}\left(\Sigma, \mathcal{I}_{C} \otimes\right.$ $\left.E_{i}(t)\right) \cong H^{0}\left(\Sigma, E_{i}(t u-a, t v-b)\right)$. Hence the integer $\operatorname{dim}\left(\operatorname{Im}\left(u_{C, t}\right)\right)$ depends only on the integers $i, t, a, b, u, v$, not on $g$ or the singularities of $C$.

Remark 6. Let $A \subset \mathbf{P}^{m}$ be a rational normal curve. Then $\Omega_{\mathbf{P}^{m}}{ }^{1}(1) \mid A$ is isomorphic to the direct sum of $m$ line bundles of degree -1 . Hence for all integers $i$ such that $0 \leq i \leq m$ the vector bundle $\Omega_{\mathbf{P}^{m}}{ }^{i}(i) \mid A$ is isomorphic to the direct sum of $\left(\begin{array}{c}m \\ i\end{array}\right)$ line bundles of degree $-i$

Remark 7. Let $V \subset \mathbf{P}^{r}$ be an $m$-dimensional linear subspace. We have $\Omega_{\mathbf{P}^{r}}^{1}{ }^{1}(1) \mid V \cong \Omega_{V}^{1}(1) \oplus \mathcal{O}_{V}^{\oplus(r-m)}$. Hence $\Omega_{\mathbf{P}^{r}}^{i}(i) \mid V \cong \bigoplus_{j=0}^{\min \{r-m, m\}} \Omega_{V}{ }^{j}(j)^{\oplus\left(\begin{array}{c}r-m \\ j\end{array}\right)}$.

Remark 8. Let $A \subset \Sigma$ be a curve of type $(1,0)$ (resp. $(0,1))$. Then $A$ is embedded in $\mathbf{P}^{r}$ as a rational normal curve of a $v$-dimensional (resp. $u$ dimensional) linear subspace. By Remarks 6 and 7 for every integer $i$ such that $0 \leq i \leq v$ (resp. $0 \leq i \leq u)$ the vector bundle $E_{i}(z, q)$ is isomorphic to the direct sum of line bundles of degree at least $q-i$ (resp. at least $z-i$ ). Hence if $i \leq \min \{u, v\}$ we have $h^{0}\left(A, E_{i}{ }^{*}(-2 u-2,-2 v-2) \mid A\right)=0$ for $A$ of one of 
the types $(1,0)$ or $(0,1)$. Hence $E_{i}(z, q) \mid A$ is spanned if $i \leq \min \{v, q\}$ (resp. $i \leq \min \{u, z\})$. Varying $A$ we get $h^{0}\left(\Sigma, E_{i}^{*}(-4)\right)=0$ for all $i \leq \max \{u, v\}$. Hence $h^{2}\left(\Sigma, E_{i}(2 u, 2 v)\right)=0$ for all $i \leq \max \{u, v\}$ (Serre duality), i.e., in this range of integers $i, u, v, a, b$ we may use Remark 5 for $t:=2$.

Remark 9. Let $D \subset \Sigma$ be an integral curve of type $(1,1)$. Hence $D \cong \mathbf{P}^{1}$ and it is embedded in $\mathbf{P}^{1}$ as a rational normal curve of an $(u+v)$-dimensional linear subspace. As in Remark 8 we see that $E_{i}(z, q) \mid D$ is isomorphic to a direct sum of line bundles of degree at most $z+q-i$ for all $0 \leq i \leq u+v$.

Proof of Theorem 3. The vector bundle $\Omega_{\mathbf{P}^{r}}{ }^{i}(i)$ is homogeneous. Since the embedding of $\Sigma$ into $\mathbf{P}^{r}$ is given by a complete embedding of an $\operatorname{Aut}^{0}(\Sigma)$ homogeneous line bundle, $h^{*}\left(E_{i}\right) \cong E_{i}$ for every $h \in \operatorname{Aut}^{0}(\Sigma)$. Since $\operatorname{char}(\mathbb{K})=$ 0 , a theorem of Matsushima implies that the vector bundle $E_{i}$ is $\operatorname{Aut}^{0}(\Sigma)$ homogeneous in the classical sense of [2]. We want to apply Bott's vanishing theorem to have $h^{1}\left(\Sigma, E_{i}(2 u-a, 2 v-b)\right)=0$ for the integers $i, a, b, u, v$ appearing in the statement of Theorem 3 and hence to get Property $N_{i}$ for $Y$. Let $D_{1} \subset \Sigma$ be a curve of type $(1,0), D_{2} \subset \Sigma$ a curve of type $(0,1)$ and $D_{3} \subset \Sigma$ an integral curve of type $(1,1)$. Hence $D \cong D_{1} \cong D_{2} \cong \mathbf{P}^{1}$ and $S L(2) \times S L(2)$ acts transitively on each curve $D_{1}, D_{2}, D_{3}$. A homogeneous vector bundle on $\Sigma$ is spanned if and only if all its direct factors are spanned. Furthermore, each of its direct factor is homogeneous. Let $A$ be an irreducible homogeneous vector bundle on $\Sigma$. By a corollary of Bott's vanishing theorem ([2], Theorem IV') $h^{i}(\Sigma, A) \neq 0$ for at most one index $i \in\{0,1,2\}$. Hence $h^{1}(\Sigma, A)=0$ if $A$ is spanned. By [7], Theorem on $\S 2$ (see its proof on p. 399 to identify the $G_{\alpha}$-orbits $\left.G_{\alpha} / B_{\alpha} \cong \mathbf{P}^{1}\right), A$ is spanned if and only if all vector bundles $A \mid D_{j}$, $j \in\{1,2,3\}$ are spanned. We apply this observation to any irreducible factor, $A$, of $E_{i}(2 u-a, 2 v-b)$. By Remarks 8 and 9 the vector bundles $E_{i}(2 u-a, 2 v-b) \mid D_{j}$, $j=1,2,3$, are spanned for all $i, u, v, a, b$ such that $0 \leq i \leq \min \{u, v, 2 v-b, 2 u-$ $a\}$.

\section{ACKNOWLEDGEMENT}

The author was partially supported by MIUR and GNSAGA of INdAM (Italy).

The author wishes to thank the referees for several very stimulating remarks.

\section{REFERENCES}

1. E. Ballico and C. Keem, Families of smooth $k$-gonal curves with another fixed pencil. Osaka J. Math. 33(1996), No. 3, 663-667.

2. R. Вотт, Homogeneous vector bundles. Ann. of Math. (2) 66(1957), 203-248; reprinted in: Collected Papers, Vol. 1, 226-248, Birkhäuser, Boston, 1994.

3. F. J. Gallego and B. P. Purnaprajna, Some results on rational surfaces and Fano varieties. J. Reine Angew. Math. 538(2001), 25-55.

4. M. Green, Koszul cohomology and the geometry of projective varieties. J. Differential Geom. 19(1984), No. 1, 125-171. 
5. D. Mumford, Varieties defined by quadratic equations. Questions on Algebraic Varieties (C.I.M.E., III Ciclo, Varenna, 1969), 29-100. Edizioni Cremonese, Rome, 1970.

6. F. Severi, Vorlesungen über Algebraische Geometrie. B. G. Teubner, Leipzig u. Berlin, 1921.

7. D. M. SNow, Spanning homogeneous vector bundles. Comment. Math. Helv. 64(1989), No. 3, 395-400.

8. A. Tannenbaum, Families of algebraic curves with nodes. Compositio Math. 41(1980), No. $1,107-126$.

9. R. Treger, Plane curves with nodes. Canad. J. Math. 41(1989), No. 2, 193-212.

(Received 6.02.2005; revised 27.07.2005)

Author's address:

Dept. of Mathematics

University of Trento

38050 Povo (TN), Italy

E-mail: ballico@science.unitn.it 